\title{
Effects of Genotype, Storage Temperature and Time on Quality and Compositional Traits of Cherry Tomato
}

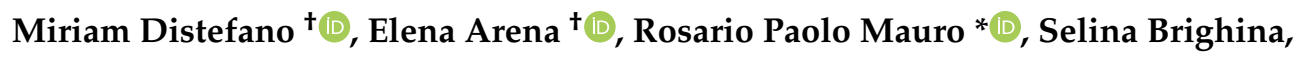 \\ Cherubino Leonardi ${ }^{\circledR}$, Biagio Fallico and Francesco Giuffrida \\ Dipartimento di Agricoltura, Alimentazione e Ambiente (Di3A), University of Catania, 95123 Catania, Italy; \\ miriam.distefano@unict.it (M.D.); earena@unict.it (E.A.); selina.brighina@unict.it (S.B.); \\ cherubino.leonardi@unict.it (C.L.); biagio.fallico@unict.it (B.F.); francesco.giuffrida@unict.it (F.G.) \\ * Correspondence: rosario.mauro@unict.it \\ + Both authors contributed equally to this work.
}

Received: 30 October 2020; Accepted: 20 November 2020; Published: 25 November 2020

\begin{abstract}
The experiment addressed the effects of two storage temperatures, namely $10\left(\mathrm{~T}_{10}\right)$ and $20^{\circ} \mathrm{C}\left(\mathrm{T}_{20}\right)$, on main quality and functional traits of three cherry tomato cultivars ('Eletta', 'Sugarland' and 'Ottymo'), after $0\left(\mathrm{~S}_{0}\right), 7\left(\mathrm{~S}_{7}\right)$ and $14\left(\mathrm{~S}_{14}\right)$ days of storage. At $\mathrm{T}_{10}$ both fruit weight and firmness were better retained during storage. At $S_{14}, T_{10}$ promoted fruit Chroma and overall fruit color deviation $\left(\Delta E_{\mathrm{ab}}^{*}\right)$. Total polyphenols content (TPC) of fruits peaked at $\mathrm{S}_{7}\left(4660 \mathrm{mg} \mathrm{GAE} \mathrm{kg}^{-1} \mathrm{DW}\right)$ then declined at $\mathrm{S}_{14}$ (by $16 \%$ ), with the highest values recorded at $\mathrm{T}_{10}$. Lycopene showed a similar trend, but with a higher average concentration recorded at $T_{20}\left(488 \mathrm{mg} \mathrm{kg}^{-1} \mathrm{DW}\right) . \beta$-carotene content peaked at $\mathrm{S}_{14}$, irrespective of the storage temperature. At $\mathrm{S}_{14}$, the concentrations of phytoene and phytofluene were higher at $\mathrm{T}_{20}$ (48.3 and $40.9 \mathrm{mg} \mathrm{kg}^{-1} \mathrm{DW}$, respectively), but the opposite was found at $\mathrm{S}_{7}$. 'Sugarland' and 'Ottymo' showed the highest $\Delta E^{*}$ ab along storage, with the former cultivar proving the highest TPC and lycopene content, whereas 'Eletta' did so for phytoene and phytofluene. Our results suggest that unravelling the possible functional interactions among these three carotenoids would allow for a better orientation of breeding programs, targeting the phytochemical evolution of tomatoes during refrigerated storage.
\end{abstract}

Keywords: Solanum lycopersicum L.; refrigerated storage; fruit quality; carotenoids; total polyphenols content

\section{Introduction}

Tomato (Solanum lycopersicum L.) is one of most important vegetable crops throughout the world, with an estimated production of $182 \mathrm{Mt}$ from more than $4.8 \mathrm{Mha}$ cropland [1]. In the Mediterranean basin it is the primary field and greenhouse vegetable crop [2], since tomato strongly characterizes the Mediterranean diet, hence its consumption is widely spread around this macroarea [3]. Fresh tomatoes commercialization is often characterized by significant temporal gaps among production and consumption. This implies the optimization of quality maintenance of the product along the distribution chain, in order to match the consumers' sensorial and nutritional demands [4]. Indeed, fresh vegetables are perishable commodities, whose postharvest decay represents a primary matter of social concern in terms of economic (loss of capital, fuel and manpower) and environmental costs (due to landfilling), associated to losses of valuable phytonutrients [5]. In this context, temperature is a key factor to extend quality of fresh horticultural products along the distribution chain [6]. Because of its sensitivity to chilling injuries [7], the optimization of tomatoes cold storage implies a compromise between temperatures low enough 
to slow down the ripening process but high enough to generate either no or tolerable side effects on the main organoleptic and nutritional traits [8]. Similarly to other plant foods [9], tomato is a source of many valuable phytonutrients having potential health benefits, including minerals, vitamins $C$ and E, organic acids, polyphenols and carotenoids [10]. Carotenoids represent by far the most studied phytochemical fraction of tomatoes [11], which are considered the main dietary source of lycopene [12], i.e., the prevailing constituent conferring the typical pigmentation to red-ripe fruits. From a nutritional viewpoint, lycopene is a powerful antioxidant, whose intake has been linked to reduced frequency and severity of several types of cancer and heart diseases [13]. Moreover, it has been indicated as the most effective singlet oxygen quencher among all known carotenoids [14]. $\beta$-carotene is the second main carotenoid constituent of tomato fruits [15]. It is a red-orange pigment having strong chemoprotective functions and the highest provitamin A activity in the human metabolism, and its deficiency can result in xerophthalmia, blindness, and even premature death [16]. Although both carotenoids can be specifically ingested through dietary supplements, scientific evidences seem to point out stronger health benefits associated to their direct assumption from tomato matrices, likely as a consequence of synergistic effects involving other naturally occurring compounds [17]. Among these, the colorless carotenoids phytoene and phytofluene have been supposed to have biological activity, as in the case of skin protection from UV-induced erythema or in the protection of human lipoproteins from oxidation [18].

Over the last decades, cherry tomato has been intensively targeted in breeding programs of many seed companies, in order to match the evolving standards in tomato production, commercialization and consumption [19]. Consequently, the currently available cultivars are characterized by better functional profile than the past [20], wide compositional variability [21] and rapid temporal turnovers. Such diversification and dynamism represent a challenging task to optimize the product management along the distribution chain, since postharvest quality modifications are strongly affected by both storage conditions and genotype [22]. Hence, to address the growing demands for tomatoes with high quality and functional profiles, it is appropriate to in-depth the knowledge about whole patterns of change in these properties, as a function of the storage conditions applied to the emerging germplasm.

Due to this, the aim of the present work was to investigate the postharvest modifications on main quality variables of three recently widespread cherry tomato cultivars in a Mediterranean environment induced by different thermal regimes $\left(10\right.$ and $20^{\circ} \mathrm{C}$ ) and storage time (up to 14 days).

\section{Materials and Methods}

\subsection{Experimental Site and Plant Material}

A greenhouse experiment was carried out from February to June 2019, at the experimental farm of the University of Catania (Sicily, South Italy: $37^{\circ} 24^{\prime} 27^{\prime \prime} \mathrm{N}, 15^{\circ} 03^{\prime} 36^{\prime \prime} \mathrm{E}, 6 \mathrm{~m}$ a.s.l.). The climate of the area is semi-arid Mediterranean, with mild winters and hot, dry summers. An $800 \mathrm{~m}^{2}$, multi-aisle cold greenhouse was used, having a steel tubular structure with adjustable windows on the roof and along the sides, and covered with polycarbonate slabs. Three cherry tomato cultivars, namely 'Eletta', 'Ottymo' and 'Sugarland', recently diffused in the reference area, were grown in the experiment, chosen on the basis of their different main carpometric traits (Table 1). To this end, data were previously acquired from different local farms operating in comparable growth conditions.

Table 1. Main information and fruit characteristics related to the cultivars selected for the study.

\begin{tabular}{cccc}
\hline & 'Eletta' & 'Ottymo' & 'Sugarland' \\
\hline Seed company & TSI Italia srl, Foggia (FG), Italy & Vilmorin Italia srl, Funo & Rijk Zwaan Italia srl, \\
Bologna (BO), Italy \\
Fruit color & Deep red & (BO), Italy & Deep red \\
Average fruit diameter $(\mathrm{mm})$ & $15 \pm 2$ & Red & $18 \pm 2$ \\
Average fruit weight $(\mathrm{g})$ & $15.0 \pm 1.5$ & $20.5 \pm 2.5$ & $12.0 \pm 1.0$ \\
\hline
\end{tabular}




\subsection{Growth Conditions, Fruit Sampling and Storage}

Plants were transplanted on 11th February 2019 within the greenhouse at the stage of two true leaves, in an open soilless cultivation system using $5 \mathrm{~L}$ plastic pots $(20 \mathrm{~cm}$ height, $19 \mathrm{~cm}$ width), with perlite as growing medium (particle size 2-6 mm). Before transplanting, plantlets were selected for uniform size and health appearance, whereas pots were arranged in simple rows, adopting a $0.40 \times 1.00 \mathrm{~m}$ rectangular format (center to center) and 1 plant per pot $\left(2.5\right.$ plants $\left.\mathrm{m}^{-2}\right)$. Plants were grown at single stem up to the 8th cluster, whereas all clusters were pruned leaving 12 fruits, whose setting was allowed by using bumblebee hives. Each net experimental unit contained 12 plants. During the cycle, the crop was uniformly fertigated with a standard nutrient solution [23], adopting a leaching fraction of at least $75 \%$, to avoid root zone salinization [24].

From 14 to 16 May, tomatoes belonging to the 4th cluster were harvested by hand at the red stage (stage F) according to Gautier et al. [25]. This was done to allow tomatoes to reach stage G (deep red) during postharvest, as it is usual among local growers. Soon after harvest, fruits were transported to the laboratory and processed for further analysis. Overall, 72 clusters were collected ( 8 clusters $\times 3$ cultivars $\times 3$ replicates) and divided in 3 batches for the characterization of fruits after 0 (harvest date), 7 and 14 days of storage (hereafter $S_{0}, S_{7}$ and $S_{14}$, respectively), either at $10 \pm 0.5\left(T_{10}\right)$ or $20 \pm 0.5{ }^{\circ} \mathrm{C}\left(\mathrm{T}_{20}\right)$ and $85 \%$ relative humidity $(\mathrm{RH})$. The lowest thermal regime was chosen since it represents a mild stressing conditions frequently adopted during transportation and storage of cherry tomatoes, whereas $T_{20}$ was comparatively chosen as it simulates storage at room temperature [26]. Before storage, fruits were detached from rachis, selected for absence of defeats and uniform appearance within each genotype, washed with deionized water and dried with paper for further analysis. Fifteen to twenty-two fruits per replicate were placed in common commercial trays, i.e., transparent PET trays Mod. C500/41p $(190 \times 115 \times 41 \mathrm{~mm})$ covered with a perforated PET LC32 lid (Carton Pack s.p.a., Rutigliano, Italy) for a final net weight of $250 \pm 8 \mathrm{~g}$, then stored at the abovementioned conditions.

\subsection{Carpometric Determinations}

At each time point, fruit fresh weight was determined on 10 fruits per tray, then their firmness was determined through a Digital Texture Analyser mod. TA-XT2 (Stable Micro Systems, Godalming, $\mathrm{UK}$ ) and defined as the force $(\mathrm{N})$ needed to impress a $2 \mathrm{~mm}$ fruit deformation along the polar axis, between two steel plates.

\subsection{Cherry Tomato Quality Variables}

For each sample, $\sim 50 \mathrm{~g}$ of cherry tomato were homogenized up to a puree in a home blender (La Moulinette, Groupe SEB, Écully, France) and immediately analyzed for: soluble solids content, dry matter, $\mathrm{pH}$, total acidity (TA), reducing sugars, total polyphenols and carotenoids profile and content.

The soluble solids content (SSC) was estimated with an Abbe refractometer 16531 (Carl Zeiss, Oberkochen, Germany) at $20^{\circ} \mathrm{C}$ and the results were expressed as ${ }^{\circ}$ Brix. The dry matter was determined by gravimetric analysis. An aliquot of cherry tomato puree were placed in an oven at $70{ }^{\circ} \mathrm{C}$ (Thermo Fisher Scientific, Waltham, MA, USA) until the constant weight [27]. The $\mathrm{pH}$ was measured using a pHmeter (Mettler Toledo, MP 220), and tritatable acidity (TA) was determined by titrating an aliquot of the puree sample with $0.05 \mathrm{~N} \mathrm{NaOH}$ to $\mathrm{pH}$ 8.1. TA was expressed as $\mathrm{g} \mathrm{kg}^{-1}$ of cherry tomato fresh weight (FW), as citric acid.

Reducing sugars (fructose and glucose), were estimated using Fehling's method according to the official Italian method of analysis (D.M. 3.2.1989, GU n.168/1989). An aliquot of the puree sample (20 g) were transferred into a volumetric flask $(50 \mathrm{~mL})$ and neutralized with $1 \mathrm{~N} \mathrm{NaOH}$. Subsequently sample was cleared by the addition of $10 \mathrm{~mL}$ saturated sodium sulphate decahydrate and $5 \mathrm{~mL}$ saturated basic lead acetate. The samples were diluted up to $50 \mathrm{~g}$ with distilled water, mixed and centrifuged for $10 \mathrm{~min}$ at 10,000 rpm. The supernatant was filtered through a filter paper (Whatman No 1, Whatman 
International, Maidstone, UK) and used to completely reduce in hot condition a mixed of the Fehling's solution using methylene blue solution as indicator. The Fehling solution was prepared as follow: $5 \mathrm{~mL}$ of each stock Fehling solution A and B were mixed with $40 \mathrm{~mL}$ of distilled water immediately before the determination. Results were expressed as g of reducing sugars $\mathrm{kg}^{-1}$ of dry weight (DW) and all analysis were conducted in triplicate.

\subsection{Fruit Chromatic Coordinates}

The fruit chromatic coordinates were measured as described by McGuire [28] on the equatorial axis of whole fruits (two measurements per fruits), through a tristimulus Minolta Chroma meter (model CR-200, Minolta Corp.) calibrated with a standard white tile (UE certificated) with illuminant D65/10 measuring color in terms of lightness $\left(L^{*}\right)$, green-red axis $\left(a^{*}\right)$ and blue-yellow axis $\left(b^{*}\right)$. Fruit color was described as $\left(a^{*} / b^{*}\right)^{2}$, Chroma [as $\left.\left(a^{* 2}+b^{* 2}\right)^{1 / 2}\right]$, tomato color index $\left[\right.$ TCI $\left.=2000 a^{*} / L^{*}\left(a^{* 2}+b^{* 2}\right)^{1 / 2}\right]$ and total color difference $\left[\Delta E_{\mathrm{ab}}^{*}=\left(\Delta L^{* 2}+\Delta a^{* 2}+\Delta b^{* 2}\right)^{1 / 2}\right]$, this last describing the color deviation recoded at $\mathrm{S}_{7}$ and $\mathrm{S}_{14}$.

\subsection{Total Polyphenols Content}

The extraction of polyphenol compounds was performed according to Atanasova et al. [29] with some modifications. An aliquot of cherry tomato puree sample $(1 \mathrm{~g})$ was mixed and shacked with $40 \mathrm{~mL}$ of acetone ( $80 \%$ solution in distilled water) and left in the dark, overnight at room temperature. After that, each sample was filtered $(0.45 \mu \mathrm{m}$ Albet $)$ and the supernatant was collected for determination of total polyphenols content (TPC). This was determined according to Gahler et al. [30] using the Folin-Ciocâlteu reagent and measuring spectrophotometrically the absorbance at $725 \mathrm{~nm}$ using a Perkin Elmer lambda $25 \mathrm{Uv}$-Vis spectrometer. Gallic acid was used as standard (standard curve, $0.29-8.18 \mathrm{mg}$ $\mathrm{kg}^{-1} ; R^{2}=1.00$ ) and TPC was expressed as mg gallic acid equivalents (GAE) $\mathrm{kg}^{-1}$ on a dry weight (DW) basis. All analyses were carried out in triplicate.

\subsection{Carotenoids Extraction and HPLC Analysis}

Tomato carotenoids were extracted using the method of Siracusa et al. [31]. An aliquot of the cherry tomato puree sample $(0.5 \mathrm{~g})$ was transferred into a vial and $5 \mathrm{~mL}$ of a n-hexane/acetone/ethanol (2:1:1) solution were added. The vial was left shaking for $40 \mathrm{~min}$. in the dark at room temperature. Subsequently $1 \mathrm{~mL}$ of $\mathrm{H}_{2} \mathrm{O}$ (HPLC grade) was added and a further $2 \mathrm{~min}$. agitation was applied. The resulting heterogeneous mixtures were left decanting until phases separation. The apolar coloured layers were transferred into an amber vial and analysed.

Quantitative analyses were carried out on an HPLC (Shimadzu USA Manufacturing Company Inc., Class VPLC-10 Dvp, Canby, OR, USA) equipped with a DAD (Shimadzu SPD-M10Avp). The column was a Gemini NX C18 $(150 \times 4.6 \mathrm{~mm} ; 3 \mu \mathrm{m}$ particle size; Phenomenex, Italy $)$, fitted with a guard cartridge packed with the same stationary phase. The flow rate was $0.7 \mathrm{~mL} / \mathrm{min}$. and the injector volume was $20 \mu \mathrm{L}$. Carotenoids were eluted with the following gradient of A (Methanol: $\mathrm{H}_{2} \mathrm{O}$ 75:25) and B (Ethyl acetate): T0 30\% B; T15 82\% B; T25 30\% B. All reagents used were HPLC purity grade: water, methanol and Ethyl acetate were obtained from Merck. The wavelength range was 220-660 nm, and the chromatograms were monitored at $473 \mathrm{~nm}$ for lycopene; at $453 \mathrm{~nm}$ for $\beta$-carotene; at $348 \mathrm{~nm}$ for phytofluene and at $288 \mathrm{~nm}$ for phytoene. Carotenoids were identified by splitting the peak of the carotenoids from the tomato-solution sample with a standard of $\beta$-carotene and lycopene; ( $p \geq 95 \%$ and $p \geq 98 \%$, Sigma-Aldrich, St. Louis, MO, USA) and by comparing retention times and UV spectra with those of standards. Quantification of $\beta$-carotene and lycopene was performed using external calibration curves; for phytofluene and phytoene the calibration curve of $\beta$-carotene was used. Linearity was checked for $\beta$-carotene between 3.36 and $21 \mathrm{mg} \mathrm{kg}^{-1}\left(R^{2}=1.00\right)$ and lycopene between 2.56 and $40.0 \mathrm{mg} \mathrm{kg}^{-1}\left(R^{2}=1.00\right)$. All analyses were performer in triplicate, including the extraction procedure, and the results were expressed as $\mathrm{mg} \mathrm{kg}^{-1} \mathrm{DW}$. 


\subsection{Statistical Procedures}

All data were subjected to Shapiro-Wilk and Levene's test, in order to check for normality and homoscedasticity, respectively, then to a factorial 'storage temperature $\times$ genotype $\times$ storage time' $(\mathrm{T} \times \mathrm{G} \times \mathrm{S})$ analysis of variance (ANOVA), according to the experimental layout adopted in the experiment. Percentage data were Bliss transformed before the ANOVA (untransformed data are reported and discussed), whereas multiple means comparisons were performed through Tukey's honestly significant difference (HSD) test $(p \leq 0.05)$. All calculations were performed using Excel version 2016 (Microsoft Corporation, Redmond, WA, USA) and Minitab version 16.1.1 (Minitab Inc., State College, PA, USA).

\section{Results}

In the present study, the significance resulting from the ANOVA related to storage temperature $(\mathrm{T})$, genotype $(G)$ and storage time $(S)$ and their first order interactions is reported in Table 2 (Fisher-Snedecor F-test), whereas their effects on variable means are reported in Tables 3-6 and Figures 1-3.

Table 2. F-test values of the main factors and their first order interactions related to observed variables, with the significance resulting from the analysis of variance.

\begin{tabular}{|c|c|c|c|c|c|c|}
\hline \multirow{2}{*}{ Variable } & \multicolumn{6}{|c|}{ Source of Variation } \\
\hline & Storage Temperature & Genotype & Storage Time & $(\mathrm{T}) \times(\mathrm{G})$ & $(\mathrm{T}) \times(\mathrm{S})$ & $(\mathrm{G}) \times(\mathrm{S})$ \\
\hline Average fruit weight & $13.6^{* * *}$ & $140.8^{* * *}$ & $65.6^{* * *}$ & $3.8^{*}$ & NS & $8.1^{* * *}$ \\
\hline Fruit dry matter & $10.6^{* *}$ & $88.4^{* * *}$ & $29.7^{* * *}$ & $3.5^{*}$ & NS & $8.7^{* * *}$ \\
\hline Fruit firmness & $8.7^{* *}$ & $29.4 * * *$ & $11.5^{* * *}$ & $3.9 *$ & NS & NS \\
\hline Reducing sugars content & NS & $50.2 * * *$ & $10.2 * * *$ & NS & NS & $3.8^{*}$ \\
\hline SSC/TA & NS & $265.2^{* * *}$ & $4.7^{*}$ & NS & NS & $12.4^{* * *}$ \\
\hline Fruit $\mathrm{pH}$ & NS & $19.6^{* * *}$ & NS & NS & NS & NS \\
\hline$\left(a^{*} / b^{*}\right)^{2}$ & NS & $38.0 * * *$ & $6.3^{* *}$ & NS & NS & NS \\
\hline Chroma & $39.4^{* * *}$ & $544.7^{* * *}$ & $30.9 * * *$ & NS & $13.2^{* * *}$ & NS \\
\hline Tomato color index & NS & $39.9 * * *$ & $4.9 *$ & NS & NS & NS \\
\hline$\Delta E_{\mathrm{ab}}^{*}$ & $8.1^{* *}$ & $15.1^{* * *}$ & $5.7 *$ & $6.0^{* *}$ & NS & $5.0 *$ \\
\hline Total polyphenols content & $20.9^{* * *}$ & $9.5^{* * *}$ & $56.4^{* * *}$ & $17.2^{* * *}$ & $4.7^{*}$ & $32.5^{* * *}$ \\
\hline Phytoene content & NS & $82.8^{* * *}$ & $7.5^{* *}$ & NS & $3.6^{*}$ & $15.1^{* * *}$ \\
\hline Phytofluene content & NS & $44.3^{* * *}$ & $6.5^{* *}$ & $6.3^{* *}$ & $3.9 *$ & $6.8^{* * *}$ \\
\hline Lycopene content & $33.8^{* * *}$ & $1462.3^{* * *}$ & $138.5^{* * *}$ & $3.6^{*}$ & $9.8^{* * *}$ & $121.4^{* * *}$ \\
\hline$\beta$-carotene content & NS & $17.4^{* * *}$ & $23.1^{* * *}$ & NS & NS & $11.7^{* * *}$ \\
\hline
\end{tabular}

SSC: soluble solids content; TA: tritatable acidity. (T): storage temperature; $(\mathrm{G})$ : genotype; $(\mathrm{S})$ : storage time. NS: not significant; ${ }^{*}, * *$ and ${ }^{* * *}$ : significant at $p \leq 0.05,0.01$ and 0.001 , respectively.

\subsection{Carpometric Traits}

Average fruit weight showed a significant ' $T \times G^{\prime}$ interaction since, passing from $T_{10}$ to $T_{20}$, 'Ottymo' and 'Sugarland' showed the highest reduction ( $-9 \%$, on average) (Table 3). Moreover, both cultivars proved the highest decline of fruit weight at the end of the storage period, as this variable was reduced by $28 \%$, on the average of both cultivars (Figure $1 \mathrm{~A}$ ).

Fruit dry matter, proved a higher value at $\mathrm{T}_{20}$ than at $\mathrm{T}_{10}$, reaching the highest rise among the thermal regimes in 'Ottymo' $(+15 \%)$ and 'Sugarland' $(+12 \%)$ (Table 3). Both genotypes highlighted the highest rise during the storage period, as their fruit dry matter increased by $44 \%$ on average, passing from $\mathrm{S}_{0}$ to $\mathrm{S}_{14}$ (Figure 1B). Differently, at $\mathrm{T}_{20}$ fruit firmness was significantly reduced, with 'Ottymo' showing the strongest decline passing from $\mathrm{T}_{10}$ to $\mathrm{T}_{20}(-19 \%)$ (Table 3). For this variable, a decreasing trend was recorded along the storage period, since, by comparison with the initial value, fruit firmness was reduced by $22 \%$ at $\mathrm{S}_{14}$ (Table 3 ). 


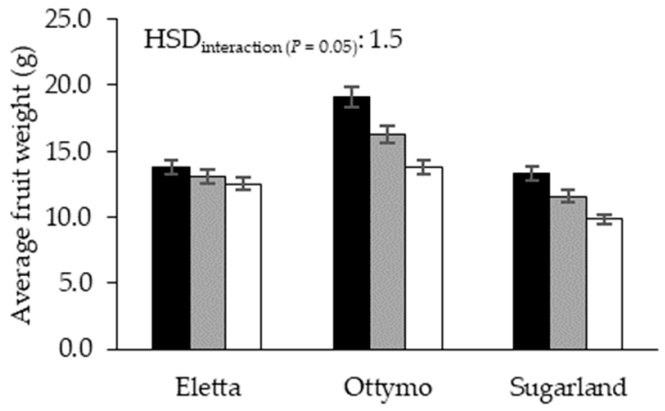

(A)

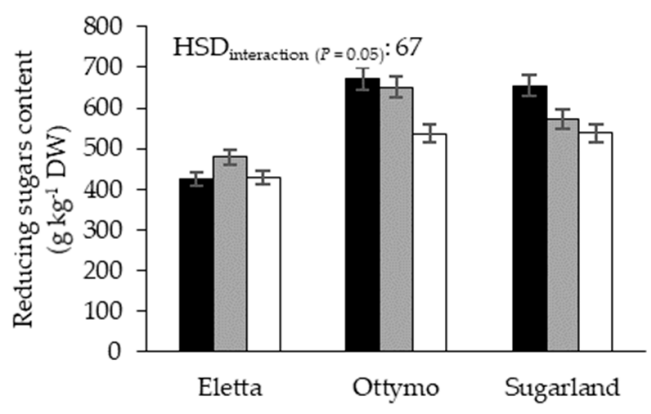

(C)

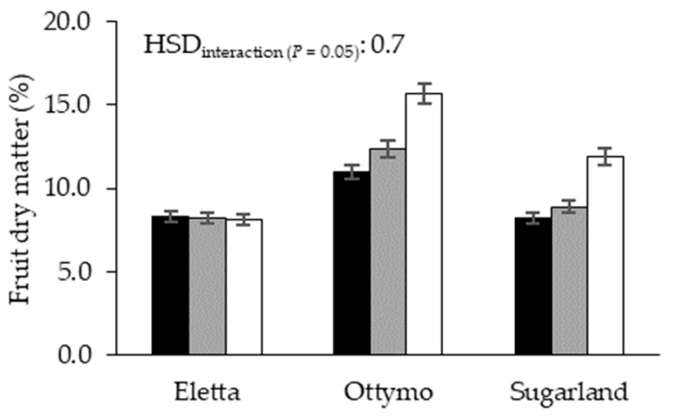

(B)

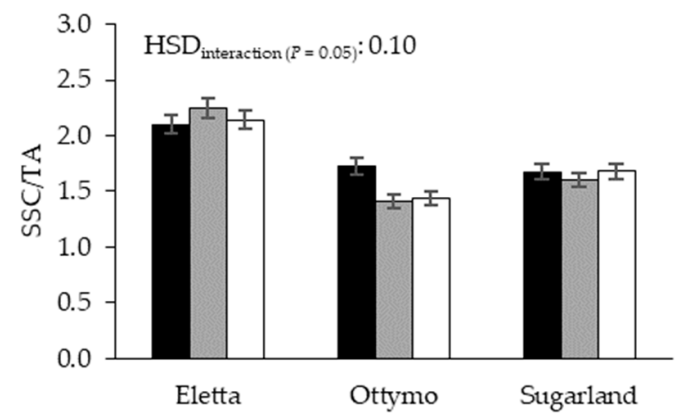

(D)

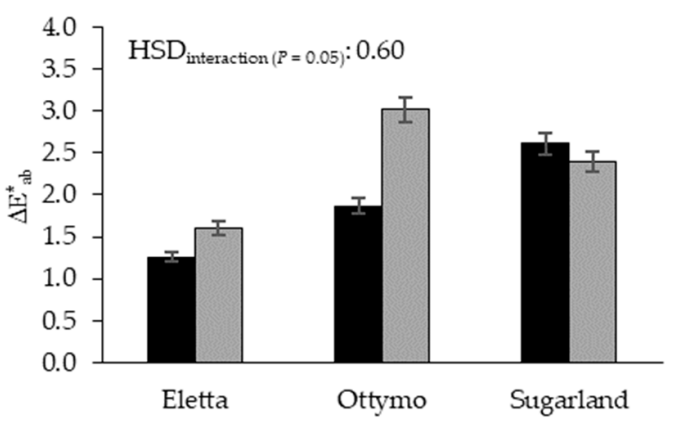

(E)

Figure 1. Average fruit weight (A), fruit dry matter (B), reducing sugars content (C), SSC/TA (D) and $\Delta E^{*}{ }_{\mathrm{ab}}(\mathrm{E})$ as affected by 'genotype $\times$ storage time' interaction. Black bars: $\mathrm{S}_{0}$; grey bars: $\mathrm{S}_{7}$; white bars $\mathrm{S}_{14}$.

Table 3. Carpometric variables of cherry tomato as affected by the main factors.

\begin{tabular}{|c|c|c|c|c|c|c|c|c|}
\hline \multirow{2}{*}{ Variable } & & \multicolumn{3}{|c|}{ Genotype } & \multicolumn{3}{|c|}{ Storage Time } & \multirow{2}{*}{$\begin{array}{c}\text { Storage Temperature } \\
\text { Mean }\end{array}$} \\
\hline & & 'Eletta' & 'Ottymo' & 'Sugarland' & $\mathrm{S}_{0}$ & $S_{7}$ & $\mathrm{~S}_{14}$ & \\
\hline \multirow{3}{*}{ Average fruit weight (g) } & $\mathrm{T}_{10}$ & 13.3 & 17.2 & 12.0 & 15.3 & 14.1 & 12.9 & $14.1^{\mathrm{a}}$ \\
\hline & $\mathrm{T}_{20}$ & 13.0 & 15.5 & 11.1 & 15.5 & 13.6 & 12.0 & $13.2^{b}$ \\
\hline & Mean & $13.2^{b}$ & $16.4^{\mathrm{a}}$ & $11.6^{\mathrm{c}}$ & $15.4^{\mathrm{a}}$ & $13.9^{b}$ & $12.5^{c}$ & \\
\hline $\mathrm{HSD}_{\text {interaction }}$ & & & 0.8 & & & 0.8 & & \\
\hline \multirow{3}{*}{$\begin{array}{l}\text { Fruit dry matter content } \\
\qquad(\%)\end{array}$} & $\mathrm{T}_{10}$ & 8.2 & 12.1 & 9.1 & 9.1 & 9.4 & 10.9 & $9.8^{\mathrm{b}}$ \\
\hline & $\mathrm{T}_{20}$ & 8.2 & 13.9 & 10.2 & 9.2 & 10.3 & 12.9 & $10.8^{\mathrm{a}}$ \\
\hline & Mean & $8.2^{c}$ & $13.0^{\mathrm{a}}$ & $9.7^{b}$ & $9.2^{b}$ & $9.8^{\mathrm{b}}$ & $11.9^{\mathrm{a}}$ & \\
\hline \multirow[t]{4}{*}{$\mathrm{HSD}_{\text {interaction }}$} & & & 1.1 & & & NS & & \\
\hline & $\mathrm{T}_{10}$ & 14.1 & 19.7 & 12.8 & 16.5 & 16.1 & 14.1 & $15.6^{\mathrm{a}}$ \\
\hline & $\mathrm{T}_{20}$ & 13.6 & 16.0 & 11.6 & 16.6 & 12.9 & 11.7 & $13.7^{\mathrm{b}}$ \\
\hline & Mean & $13.8^{\mathrm{b}}$ & $17.9^{\mathrm{a}}$ & $12.2^{\mathrm{c}}$ & $16.6^{\mathrm{a}}$ & $14.5^{\mathrm{b}}$ & $12.9^{c}$ & \\
\hline $\mathrm{HSD}_{\text {interaction }}$ & & & 2.2 & & & NS & & \\
\hline
\end{tabular}

Different letters among factor means indicate significance at Tukey's HSD test $(p \leq 0.05)$. Interaction values $(p=0.05)$ related to 'storage temperature $\times$ genotype' and 'storage temperature $\times$ storage time' are reported. NS: not significant. 


\subsection{Cherry Tomato Quality Variables}

On the average of the other factors, 'Ottymo' and 'Sugarland' proved the highest reducing sugars content (581 $\mathrm{g} \mathrm{kg}^{-1} \mathrm{DW}$, on average), whereas the former cultivar proved the lowest $\mathrm{pH}$; differently, 'Sugarland' minimized the SSC/TA ratio (Table 4). Both reducing sugars content and SSC/TA declined passing from $\mathrm{S}_{0}$ to $\mathrm{S}_{14}$ (by 14 and $4 \%$, respectively). For the former variable, the strongest reduction along the storage period was noticed in 'Ottymo' and 'Sugarland' ( $-19 \%$, on average) (Figure 1C), whereas for SSC/TA the only significant reduction within the $\mathrm{S}_{0}-\mathrm{S}_{14}$ period was found in 'Ottymo' $(-17 \%)$ (Figure 1D).

Table 4. Compositional variables related to fruit taste of cherry tomato as affected by the main factors.

\begin{tabular}{|c|c|c|c|c|c|c|c|c|}
\hline \multirow{2}{*}{ Variable } & & \multicolumn{3}{|c|}{ Genotype } & \multicolumn{3}{|c|}{ Storage Time } & \multirow{2}{*}{$\begin{array}{c}\text { Storage Temperature } \\
\text { Mean }\end{array}$} \\
\hline & & 'Eletta' & 'Ottymo' & 'Sugarland' & $S_{0}$ & $\mathrm{~S}_{7}$ & $\mathrm{~S}_{14}$ & \\
\hline \multirow{3}{*}{$\begin{array}{l}\text { Reducing sugars content } \\
\qquad\left(\mathrm{g} \mathrm{kg}^{-1} \mathrm{DW}\right)\end{array}$} & $\mathrm{T}_{10}$ & 434 & 595 & 552 & 557 & 555 & 463 & $527^{a}$ \\
\hline & $\mathrm{T}_{20}$ & 420 & 597 & 579 & 565 & 536 & 499 & $529^{a}$ \\
\hline & Mean & $427^{b}$ & $596^{\mathrm{a}}$ & $565^{a}$ & $561^{a}$ & $545^{\mathrm{a}}$ & $481^{b}$ & \\
\hline $\mathrm{HSD}_{\text {interaction }}$ & & & NS & & & NS & & \\
\hline \multirow{3}{*}{$\begin{array}{c}\text { SSC/TA } \\
\text { (adimensional) }\end{array}$} & $\mathrm{T}_{10}$ & 2.15 & 1.65 & 1.52 & 1.80 & 1.77 & 1.71 & $1.77^{\mathrm{a}}$ \\
\hline & $\mathrm{T}_{20}$ & 2.18 & 1.66 & 1.54 & 1.86 & 1.74 & 1.80 & $1.79^{\mathrm{a}}$ \\
\hline & Mean & $2.16^{\mathrm{a}}$ & $1.65^{b}$ & $1.53^{c}$ & $1.83^{\mathrm{a}}$ & $1.76^{b}$ & $1.76^{\mathrm{b}}$ & \\
\hline \multirow{2}{*}{$\mathrm{HSD}_{\text {interaction }}$} & & & NS & & & NS & & \\
\hline & $\mathrm{T}_{10}$ & 4.59 & 4.21 & 4.33 & 4.37 & 4.35 & 4.42 & $4.38^{\mathrm{a}}$ \\
\hline \multirow[t]{2}{*}{ Fruit $\mathrm{pH}$} & $\mathrm{T}_{20}$ & 4.61 & 4.22 & 4.38 & 4.38 & 4.42 & 4.42 & $4.40^{\mathrm{a}}$ \\
\hline & Mean & $4.60^{\mathrm{a}}$ & $4.21^{\mathrm{b}}$ & $4.36^{\mathrm{ab}}$ & $4.37^{\mathrm{a}}$ & $4.38^{a}$ & $4.42^{\mathrm{a}}$ & \\
\hline $\mathrm{HSD}_{\text {interaction }}$ & & & NS & & & NS & & \\
\hline
\end{tabular}

Different letters among factor means indicate significance at Tukey's HSD test $(p \leq 0.05)$. Interaction values $(p=0.05)$ related to 'storage temperature $\times$ genotype' and 'storage temperature $\times$ storage time' are reported. NS: not significant.

\subsection{Chromatic Variables}

Among the chromatic variables, Chroma and $\Delta E^{*}$ ab showed a similar response to storage temperature, as they were both increased at $\mathrm{T}_{10}$ (by $4 \%$ and $27 \%$, respectively) (Table 5 ). For Chroma, the increase under cold storage was particularly evident passing from $S_{7}(24.1)$ to $S_{14}(26.0,+8 \%)$ (Table 5). Among the studied genotypes, 'Eletta' showed the highest $\left(a^{*} / b^{*}\right)^{2}$ and Chroma $(0.81$ and 26.4, respectively) and the lowest $\Delta E_{\mathrm{ab}}^{*}(1.43)$, whereas the lowest TCI was found in 'Sugarland' (31.3) (Table 5). All the chromatic variables significantly increased between $S_{7}$ and $S_{14}$, but for $\Delta E^{*}$ ab such temporal rise was more prominent in 'Ottymo' (by 61\%) (Figure 1E).

Table 5. Chromatic variables of the epicarp of cherry tomato as affected by the main factors.

\begin{tabular}{|c|c|c|c|c|c|c|c|c|}
\hline \multirow{2}{*}{\multicolumn{2}{|c|}{ Variable }} & \multicolumn{3}{|c|}{ Genotype } & \multicolumn{3}{|c|}{ Storage Time } & \multirow{2}{*}{ Storage Temperature Mean } \\
\hline & & 'Eletta' & 'Ottymo' & 'Sugarland' & $\mathrm{S}_{0}$ & $S_{7}$ & $\mathrm{~S}_{14}$ & \\
\hline \multirow{3}{*}{$\left(a^{*} / b^{*}\right)^{2}$} & $\mathrm{~T}_{10}$ & 0.79 & 0.74 & 0.59 & 0.72 & 0.67 & 0.73 & $0.71^{\mathrm{a}}$ \\
\hline & $\mathrm{T}_{20}$ & 0.84 & 0.71 & 0.63 & 0.72 & 0.67 & 0.78 & $0.73^{\mathrm{a}}$ \\
\hline & Mean & $0.81^{\mathrm{a}}$ & $0.73^{b}$ & $0.61^{\mathrm{c}}$ & $0.72^{\mathrm{a}}$ & $0.67^{\mathrm{b}}$ & $0.75^{\mathrm{a}}$ & \\
\hline \multicolumn{2}{|c|}{$\mathrm{HSD}_{\text {interaction }}$} & & NS & & & NS & & \\
\hline & $\mathrm{T}_{10}$ & 26.8 & 26.5 & 21.1 & 24.5 & 24.1 & 26.0 & $24.8^{\mathrm{a}}$ \\
\hline \multirow[t]{2}{*}{ Chroma } & $\mathrm{T}_{20}$ & 26.1 & 25.2 & 20.3 & 24.3 & 23.1 & 24.1 & $23.8^{\mathrm{b}}$ \\
\hline & Mean & $26.4^{\mathrm{a}}$ & $25.9^{\mathrm{b}}$ & $20.7^{c}$ & $24.4^{\mathrm{b}}$ & $23.6^{\mathrm{c}}$ & $25.1^{\mathrm{a}}$ & \\
\hline \multicolumn{2}{|c|}{$\mathrm{HSD}_{\text {interaction }}$} & & NS & & & 0.7 & & \\
\hline \multirow{3}{*}{ TCI } & $\mathrm{T}_{10}$ & 33.9 & 34.9 & 31.1 & 33.1 & 32.9 & 33.8 & $33.3^{\mathrm{a}}$ \\
\hline & $\mathrm{T}_{20}$ & 34.1 & 34.2 & 31.6 & 33.1 & 32.6 & 34.0 & $33.3^{\mathrm{a}}$ \\
\hline & Mean & $34.0^{\mathrm{a}}$ & $34.5^{\mathrm{a}}$ & $31.3^{\mathrm{b}}$ & $\begin{array}{c}33.1 \\
\mathrm{ab}\end{array}$ & $32.7^{\mathrm{b}}$ & $33.9^{\mathrm{a}}$ & \\
\hline \multicolumn{2}{|c|}{$\mathrm{HSD}_{\text {interaction }}$} & & NS & & & NS & & \\
\hline \multirow{3}{*}{$\Delta E_{\mathrm{ab}}^{*}$} & $\mathrm{~T}_{10}$ & 1.37 & 2.65 & 3.11 & - & 2.18 & 2.57 & $2.38^{\mathrm{a}}$ \\
\hline & $\mathrm{T}_{20}$ & 1.49 & 2.36 & 1.77 & - & 1.64 & 2.10 & $1.87^{\mathrm{b}}$ \\
\hline & Mean & $1.43^{\mathrm{b}}$ & $2.50^{\mathrm{a}}$ & $2.44^{\mathrm{a}}$ & - & $1.91^{\mathrm{b}}$ & $2.34^{\mathrm{a}}$ & \\
\hline \multicolumn{2}{|c|}{$\mathrm{HSD}_{\text {interaction }}$} & & 0.6 & & & NS & & \\
\hline
\end{tabular}

Different letters among factor means indicate significance at Tukey's HSD test $(p \leq 0.05)$. Interaction values $(p=0.05)$ related to 'storage temperature $\times$ genotype' and 'storage temperature $\times$ storage time' are reported. NS: not significant; -: no data. 


\subsection{Total Polyphenols Content}

Total polyphenols content (TPC) was significantly higher at $\mathrm{T}_{10}\left(4327 \mathrm{mg} \mathrm{GAE} \mathrm{kg}^{-1} \mathrm{DW}\right)$ that at $\mathrm{T}_{20}$ (4034 mg GAE kg-1 DW) (Table 6), but with strong interactive effects with genotype and storage time. Indeed, while 'Eletta' showed no differences among the 2 thermal regimes, TPC was strongly promoted by the lowest thermal regime in 'Sugarland' $(+20 \%)$, followed by 'Ottymo' $(+9 \%)$ (Table 6$)$. As regards its temporal trend, TPC significantly increased passing from $S_{0}$ to $S_{7}(+17 \%)$ then sharply declined at $\mathrm{S}_{14}(-16 \%)$, with a steeper rise in the $\mathrm{S}_{0}-\mathrm{S}_{7}$ period recorded at $\mathrm{T}_{10}(+22 \%)$ than at $\mathrm{T}_{20}(+12 \%)$ (Table 6). Moreover, the studied genotypes displayed different time-courses of TPC along the storage period, since 'Sugarland' proved the highest TPC rise passing from $S_{0}$ to $S_{7}(+37 \%)$ followed by the strongest decline at $\mathrm{S}_{14}(-33 \%)$ (Figure $\left.3 \mathrm{~A}\right)$.

\subsection{Carotenoids Content}

Figure 2 shows the HPLC carotenoids profile extracted from cherry tomato 'Sugarland'. At harvest date, the level of lycopene ranged from 68.1 to $582.5 \mathrm{mg} \mathrm{kg}^{-1} \mathrm{DW}$ in 'Ottymo' and 'Sugarland', respectively, followed by $\beta$-carotene, which varied from 72.8 to $82.17 \mathrm{mg} \mathrm{kg}^{-1} \mathrm{DW}$, in 'Ottymo and 'Eletta', respectively. Among the genotype tested, 'Eletta' proved the highest levels of both phytoene and phytofluene (54.2 and $50.7 \mathrm{mg} \mathrm{kg}^{-1} \mathrm{DW}$, respectively). The levels determined in 'Sugarland' and 'Ottymo' varied from 31.0 to $38.2 \mathrm{mg} \mathrm{kg}^{-1} \mathrm{DW}$, for phytoene and from 36.1 to $39.9 \mathrm{mg} \mathrm{kg}^{-1} \mathrm{DW}$, for phytofluene, respectively.

The phytoene content of the studied genotypes proved different time courses among the 2 thermal regimes, as it significantly increased passing from $S_{7}$ to $S_{14}$ when the $T_{20}$ storage was considered (from 41.2 to $48.3 \mathrm{mg} \mathrm{kg}^{-1} \mathrm{DW},+14 \%$ ) (Table 6). Among the genotypes, 'Sugarland' proved the highest phytoene rise passing from $\mathrm{S}_{0}$ to $\mathrm{S}_{7}$ (from 36.2 to $46.1 \mathrm{mg} \mathrm{kg}^{-1} \mathrm{DW},+28 \%$ ), whereas in 'Ottymo' a significant increase was recorded between $S_{7}\left(35.1 \mathrm{mg} \mathrm{kg}^{-1} \mathrm{DW}\right)$ and $\mathrm{S}_{14}\left(45.3 \mathrm{mg} \mathrm{kg}^{-1} \mathrm{DW},+29 \%\right)$ (Figure 3B).

Table 6. Nutraceutical variables of cherry tomato as affected by the main factors.

\begin{tabular}{|c|c|c|c|c|c|c|c|c|}
\hline \multirow{2}{*}{ Variable } & & \multicolumn{3}{|c|}{ Genotype } & \multicolumn{3}{|c|}{ Storage Time } & \multirow{2}{*}{$\begin{array}{c}\text { Storage Temperature } \\
\text { Mean }\end{array}$} \\
\hline & & 'Eletta' & 'Ottymo' & 'Sugarland' & $\mathrm{S}_{0}$ & $\mathrm{~S}_{7}$ & $\mathrm{~S}_{14}$ & \\
\hline \multirow{3}{*}{$\begin{array}{c}\text { TPC } \\
\left(\mathrm{mg} \mathrm{GAE} \mathrm{kg}^{-1} \mathrm{FW}\right)\end{array}$} & $\mathrm{T}_{10}$ & 4087 & 4531 & 4364 & 3997 & 4869 & 4116 & $4327^{a}$ \\
\hline & $\mathrm{T}_{20}$ & 4287 & 4167 & 3647 & 3982 & 4452 & 3668 & $4034^{b}$ \\
\hline & Mean & $4187^{b}$ & $4349^{a}$ & $4006^{c}$ & $\begin{array}{c}3989 \\
\text { b }\end{array}$ & $\begin{array}{c}4660 \\
a\end{array}$ & $\begin{array}{c}3892 \\
\mathrm{~b}\end{array}$ & \\
\hline $\mathrm{HSD}_{\text {interaction }}$ & & & 303 & & & 303 & & \\
\hline \multirow{3}{*}{$\begin{array}{l}\text { Phytoene content } \\
\left.\text { (mg kg }{ }^{-1} \mathrm{FW}\right)\end{array}$} & $\mathrm{T}_{10}$ & 50.4 & 41.5 & 41.2 & 43.3 & 45.5 & 45.1 & $44.3^{\mathrm{a}}$ \\
\hline & $\mathrm{T}_{20}$ & 53.5 & 38.5 & 41.3 & 43.1 & 41.2 & 48.3 & $44.5^{\mathrm{a}}$ \\
\hline & Mean & $51.9^{\mathrm{a}}$ & $40.0^{\mathrm{b}}$ & $41.2^{b}$ & $43.2^{b}$ & $43.3^{b}$ & $46.7^{\mathrm{a}}$ & \\
\hline $\mathrm{HSD}_{\text {interaction }}$ & & & NS & & & 3.2 & & \\
\hline \multirow{3}{*}{$\begin{array}{l}\text { Phytofluene content } \\
\qquad\left(\mathrm{mg} \mathrm{kg}^{-1} \mathrm{FW}\right)\end{array}$} & $\mathrm{T}_{10}$ & 42.1 & 38.7 & 32.3 & 39.6 & 37.7 & 35.6 & $37.7^{\mathrm{a}}$ \\
\hline & $\mathrm{T}_{20}$ & 46.5 & 34.6 & 33.9 & 39.8 & 33.4 & 41.9 & $38.3^{a}$ \\
\hline & Mean & $44.3^{\mathrm{a}}$ & $36.6^{b}$ & $33.1^{\mathrm{c}}$ & $39.7^{\mathrm{a}}$ & $35.5^{c}$ & $38.8^{b}$ & \\
\hline $\mathrm{HSD}_{\text {interaction }}$ & & & 3.5 & & & 3.5 & & \\
\hline \multirow{3}{*}{$\begin{array}{l}\text { Lycopene content } \\
\left(\mathrm{mg} \mathrm{kg}^{-1} \mathrm{FW}\right)\end{array}$} & $\mathrm{T}_{10}$ & 488 & 185 & 662 & 392 & 526 & 404 & $445^{b}$ \\
\hline & $\mathrm{T}_{20}$ & 556 & 207 & 701 & 416 & 577 & 484 & $488^{a}$ \\
\hline & Mean & $552^{b}$ & $196^{c}$ & $682^{a}$ & $404^{c}$ & $552^{a}$ & $444^{\mathrm{b}}$ & \\
\hline \multirow{4}{*}{$\begin{array}{l}\mathrm{HSD}_{\text {interaction }} \\
\beta \text {-carotene content } \\
\left(\mathrm{mg} \mathrm{kg}^{-1} \mathrm{DW}\right)\end{array}$} & & & 35 & & & 35 & & \\
\hline & $\mathrm{T}_{10}$ & 92.0 & 85.2 & 83.4 & 77.0 & 89.7 & 94.0 & $86.9^{a}$ \\
\hline & $\mathrm{T}_{20}$ & 96.0 & 81.7 & 77.1 & 76.7 & 85.9 & 92.3 & $84.9^{\mathrm{a}}$ \\
\hline & Mean & $94.0^{\mathrm{a}}$ & $83.5^{b}$ & $80.3^{b}$ & $76.8^{c}$ & $87.8^{b}$ & $93.1^{\mathrm{a}}$ & \\
\hline $\mathrm{HSD}_{\text {interaction }}$ & & & NS & & & NS & & \\
\hline
\end{tabular}

Different letters among factor means indicate significance at Tukey's HSD test $(p \leq 0.05)$. Interaction values $(p=0.05)$ related to 'storage temperature $\times$ genotype' and 'storage temperature $\times$ storage time' are reported. NS: not significant. 

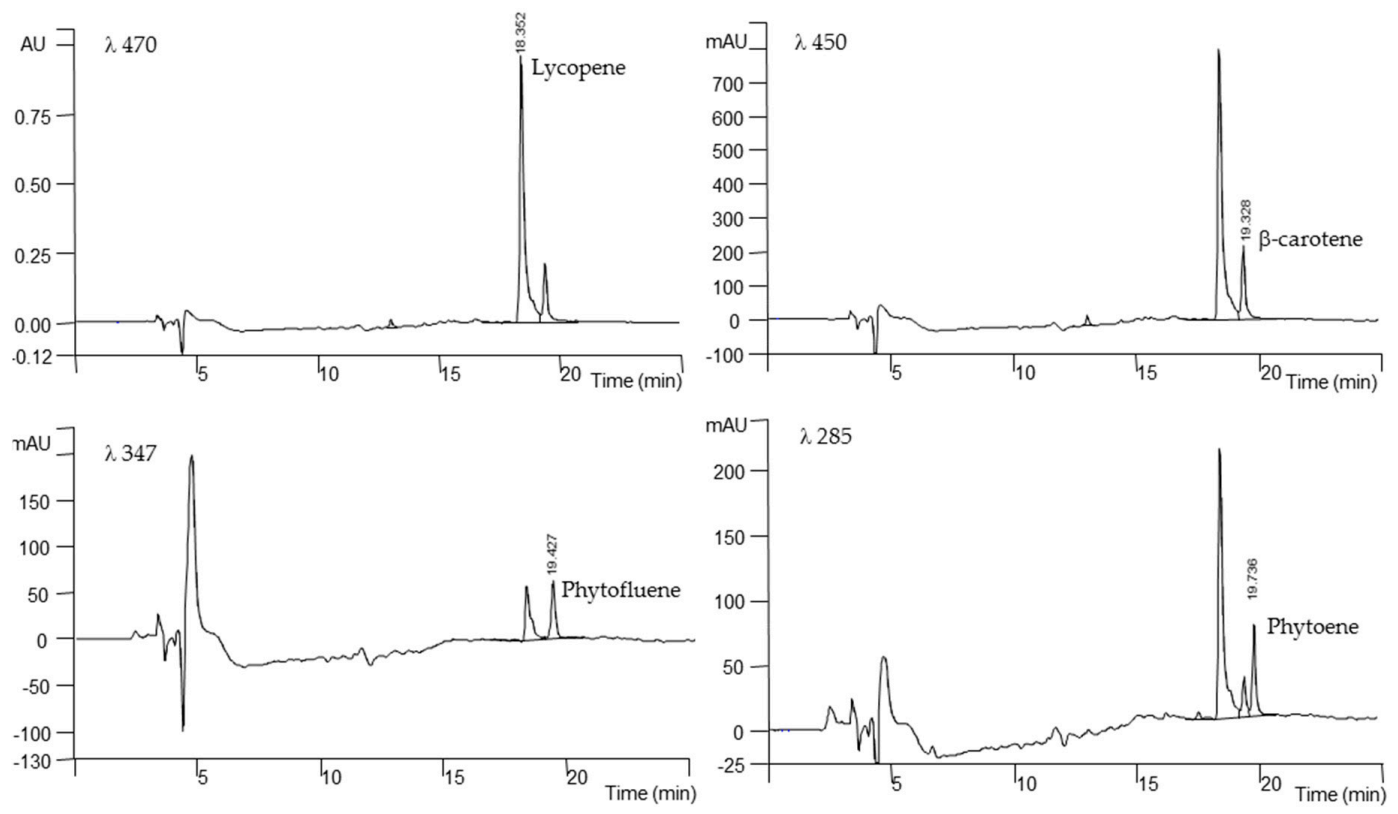

Figure 2. HPLC profile of carotenoids extracted from cherry tomato 'Sugarland' at harvest date $\left(\mathrm{S}_{0}\right)$.

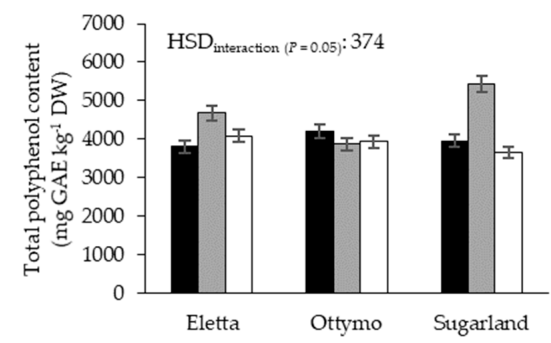

(A)

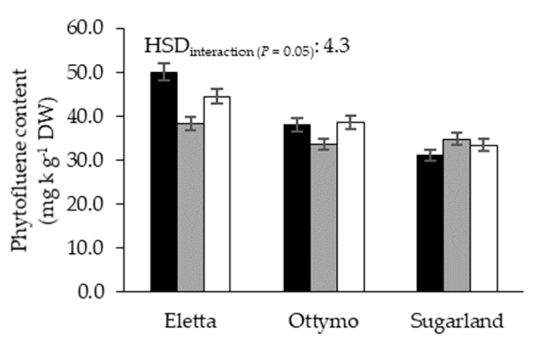

(C)

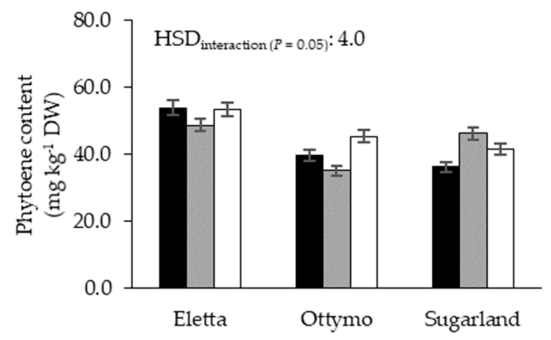

(B)

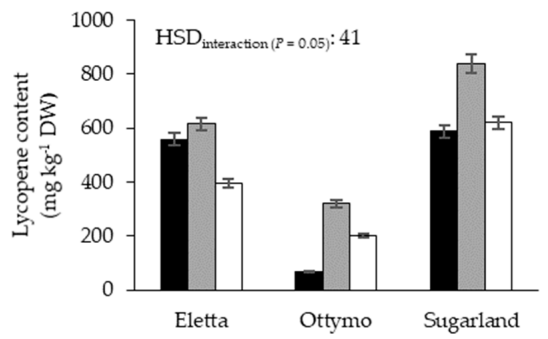

(D)

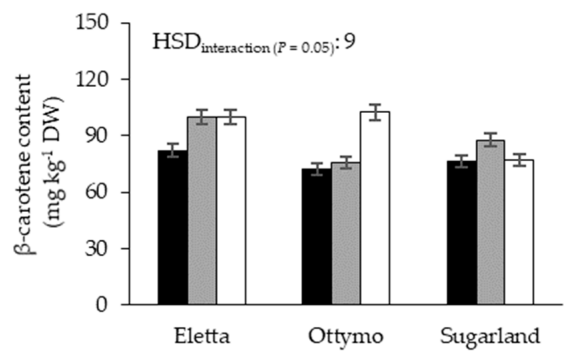

(E)

Figure 3. Total polyphenols (A), phytoene (B), phytofluene (C), lycopene (D) and $\beta$-carotene (E) content as affected by 'genotype $\times$ storage time' interaction. Black bars: $\mathrm{S}_{0}$; grey bars: $\mathrm{S}_{7}$; white bars $\mathrm{S}_{14}$. 
Regarding phytofluene, the lowest storage temperature showed a depressive effect in 'Eletta' (in which it was reduced by 9\%) and the opposite in 'Ottymo' (in which it increased by 10\%) (Table 6). Phytofluene content proved also wider temporal oscillations at $\mathrm{T}_{20}$, as the initial value was reduced by $6.4 \mathrm{mg} \mathrm{kg}^{-1} \mathrm{DW}$ at $\mathrm{S}_{7}(-9 \%)$, then increased by $6.4 \mathrm{mg} \mathrm{kg}^{-1} \mathrm{DW}$ at $\mathrm{S}_{14}(+19 \%)$ (Table 6$)$. Such temporal oscillations proved to be genotype-dependent too, since 'Eletta' showed the highest reduction passing from $\mathrm{S}_{0}\left(50 \mathrm{mg} \mathrm{kg}^{-1} \mathrm{DW}\right)$ to $\mathrm{S}_{7}\left(38.4 \mathrm{mg} \mathrm{kg}^{-1} \mathrm{DW},-23 \%\right)$, then the sharpest rise at $\mathrm{S}_{14}\left(44.5 \mathrm{mg} \mathrm{kg}^{-1}\right.$ DW, $+16 \%$ ) (Figure 3C).

Lycopene was significantly affected by the storage temperature, as it was lower at $T_{10}$ than at $T_{20}$ ( 445 vs. $488 \mathrm{mg} \mathrm{kg}^{-1} \mathrm{DW}$ ), and this reduction was more marked for 'Eletta' $(-12 \%)$ and 'Sugarland' $(-6 \%)$ (Table 6). Moreover, $\mathrm{T}_{20}$ promoted a sharper lycopene rise than $\mathrm{T}_{10}$ passing from $\mathrm{S}_{0}$ to $\mathrm{S}_{7}$ (from 416 to $577 \mathrm{mg} \mathrm{kg}^{-1} \mathrm{DW},+39 \%$ ) followed by a milder decrease at $\mathrm{S}_{14}\left(484 \mathrm{mg} \mathrm{kg}^{-1} \mathrm{DW},-16 \%\right)$ (Table 6). All the studied cultivars showed a significant decrease in lycopene content between $\mathrm{S}_{7}$ and $\mathrm{S}_{14}$ (ranging from 119 to $221 \mathrm{mg} \mathrm{kg}^{-1} \mathrm{DW}$ in 'Ottymo and 'Eletta', respectively), with 'Ottymo' and 'Sugarland' proving also a higher lycopene increase between $S_{0}$ and $S_{7}$ (by $252 \mathrm{mg} \mathrm{kg}^{-1} \mathrm{DW}$, on average) (Figure 3D).

$\beta$-carotene concentration proved to be not sensitive to the storage temperature, and was higher in 'Eletta' (94.0 $\left.\mathrm{mg} \mathrm{kg}^{-1} \mathrm{DW}\right)$ than in the other genotypes $\left(81.9 \mathrm{mg} \mathrm{kg}^{-1} \mathrm{DW}\right.$, on average) and, over the storage period, increased up to $93.1 \mathrm{mg} \mathrm{kg}^{-1} \mathrm{DW}$ at $\mathrm{S}_{14}$ (Table 6). However, such temporal increase was more marked in 'Eletta' within the $\mathrm{S}_{0}-\mathrm{S}_{7}$ period (from 82.1 to $100.0 \mathrm{mg} \mathrm{kg}^{-1} \mathrm{DW}$ ) and in 'Ottymo' in the $S_{7}-S_{14}$ one (from 75.9 to $102.3 \mathrm{mg} \mathrm{kg}^{-1} \mathrm{DW}$ ) (Figure 3E).

\section{Discussion}

The fruits stored at $10{ }^{\circ} \mathrm{C}$ showed a higher fruit weight and a lower dry matter content as compared to those stored at $20^{\circ} \mathrm{C}$, indicating that fruit transpiration and water loss were the main processes affected by storage temperature. As a consequence, at $20{ }^{\circ} \mathrm{C}$ tomatoes proved a higher loss of fruit firmness over time. The transpiration-driven softening of tomatoes during postharvest is a major problem, as it increases their susceptibility to damages along the distribution chain [32]. Moreover, fruit firmness is considered a key indicator of tomato freshness, able to influence the purchasing behavior of consumers [33]. However, despite cold storage is commonly practiced for reducing postharvest softening of tomatoes, the opposite effect can be found when too low storage temperatures are used, because of the tropical origin of the plant [8]. For this reason, storage temperature over $11-12{ }^{\circ} \mathrm{C}$ are advised for storing tomatoes, depending on fruit typology and ripening stage [32-35]. Nonetheless, the differences in terms of fruit weight and firmness we found among the 2 thermal regimes showed that storage at $10^{\circ} \mathrm{C}$ was a suitable way to extend these main characteristics of tomato fruits. Among the studied cultivars, both 'Sugarland' (small-fruited) and 'Ottymo' (large-fruited) showed the highest fruit weight reduction during storage, consistent with their steeper rise in dry matter content. Differently, 'Eletta' (medium-fruited) proved the highest temporal stability in relation to both variables. Hence our results suggest that the genotypic attitude of cherry tomato to retain fruit weight and firmness during postharvest, is dependent from factors other than simply the fruit size (i.e., the ratio among berry volume and its external transpiring surface) [36], and likely due to the functional traits of the epicarp. Indeed, it has been reported that the dynamics of fruit water loss and consequent tissue collapse are influenced by genotypic differences in structural characteristics of the cuticle, whose alteration over time is an intrinsic feature of the genetically-programmed ripening process [37].

In tomato, the ethylene-driven ripening and senescence lead to the alteration of the carbon substrates content [38], as they are energy-requiring processes whose kinetic is influenced by the ambient temperature [24]. In our experiment, reducing sugars content, the ratio SSC/TA and fruit $\mathrm{pH}$ were not affected by the storage temperature, proving instead to be genotype-dependent. Despite their higher increase in dry matter, 'Sugarland' and 'Ottymo' highlighted the steepest drop in reducing sugars content at the end of storage period (by $19 \%$, on average), denoting within the $10-20{ }^{\circ} \mathrm{C}$ range 
a temperature-insensitive acceleration of their autocatalytic metabolism. This demonstrates that no chilling disturbance in reducing sugars metabolism occurred in the experiment [34]. To this end, while the cultivars did not show appreciable $\mathrm{pH}$ variations during storage, 'Ottymo' proved the highest SSC/TA reduction over time, denoting its lowest suitability to keep unchanged the taste peculiarities. Indeed, the SSC/TA ratio is a pivotal organoleptic descriptor, as it is related to the overall balance in the perceived sweetness (SSC) and sourness (TA) of tomatoes [39].

Color is one of most important and widely used parameters to define the quality of tomato and tomato products [40]. When fresh tomato fruits are concerned, it is linked to fruit ripeness and firmness and is generally associated by consumers to tomatoes eating quality. In the present experiment, we used an array of chromatic variables summarizing the main color modifications occurring in tomato epicarp. Chroma, $\left(a^{*} / b^{*}\right)^{2}$ and tomato color index have been related to quality traits of tomato [41,42], whereas $\Delta E^{*}$ ab has been successfully used to monitor the quality maintenance of potato sticks during refrigerated storage [43]. All these variables showed a certain variability among the studied cultivars, with two of them, namely Chroma and $\Delta E_{\mathrm{ab}}^{*}$, increasing at $\mathrm{T}_{10}$, overall indicating a higher deviation toward more vivid fruit colors. In particular, after 14 days of storage, a higher reduction of Chroma was recorded at $20^{\circ} \mathrm{C}$, a condition which matched the strongest decrease in fruit weight and firmness experienced by the studied cultivars. 'Sugarland' and 'Ottymo' proved higher $\Delta E^{*}$ ab variations during storage. According to Dattner and Bohn [44], independently from the deviation formula, two colors can be optically distinguished if $\Delta E>1$. The $\Delta E^{*}$ ab differences attained by 'Sugarland' and 'Ottymo' (2.47 units, on average) and 'Eletta' (1.43) indicate for the former cultivars a higher perceivable color deviation along the storage period, consistent with their higher qualitative decline in terms of fruit weight and turgor.

When phytochemical composition was concerned, total polyphenols, lycopene and $\beta$-carotene contents found in our experiment were substantially in line with those reported by Fernandes et al. [45] for cherry tomato 'Moscatel RZ' grown in hydroponic or semi-hydroponic systems. On the other hand, phytoene and phytofluene contents were very similar to those found in cherry tomato by Mapelli-Brahm et al. [46]. Plant polyphenols are a large group of phytochemicals involved in the regulation of plant growth, reproduction and response to the environmental stressors [47]. From a nutraceutical viewpoint, they have strong antioxidant properties probably implicated in the decreased incidence of cardiovascular diseases and certain forms of cancer [48]. Both thermal regimes promoted a bell-shaped postharvest trend of TPC, consisting in their sharp rise at $S_{7}$, followed by a decrease at $\mathrm{S}_{14}$, this last indicating the onset of metabolic senescence processes [49]. However, such increase was more marked at $10{ }^{\circ} \mathrm{C}$, suggesting the occurrence of a cold-adaptive response in up-regulating the polyphenols expression during postharvest storage. Indeed, several phenolic compounds typically accumulate in plant cells subjected to cold stress, as they contribute to the homeostasis of cold-induced reactive oxygen species (ROS) and to enhance the thickness of the cell wall, so preventing lipid peroxidation and cell collapse [47]. This would explain the best retention of fruit firmness recorded at $10{ }^{\circ} \mathrm{C}$, indicating at the same time, the improvement of tomato phenolic profile as a benefit induced by a mild cold stress. Thus, although polyphenols have not been considered a priority target in tomato breeding programs, our results suggest that they could represent a sensitive target for improving the functional profile of the tomato, mostly during postharvest cold storage.

Regarding the carotenoid fraction, we recorded variable effects, resulting from different time-course response to storage temperature and duration. Lycopene displayed a bell-shaped temporal trend too since, under both storage temperatures, this carotenoid sharply increased at $S_{7}$ then declined at $S_{14}$. This trend substantially differed from that of $\beta$-carotene which continuously increased until $\mathrm{S}_{14}$, so confirming the higher stability of its postharvest accumulation in tomato [50]. According to Rodriguez-Amaya [51], carotenoids accumulation can continue during postharvest transport or storage, provided that the integrity of the fruit is maintained, so preserving the enzymatic activity responsible for carotenogenesis. Lycopene plays a paramount function in protecting the photosynthetic apparatus and plant lipid membranes, as its acyclic polyene structure (11 conjugated double bonds) increases its 
affinity for singlet oxygen and radical scavenging activity beyond the other carotenoids [52]. For this reason, it has been reported that oxidation is the main cause for lycopene degradation [14]. This could partly explain the depressive effect on lycopene content we recorded upon storing tomatoes in a stressing, ROS-inducing environment $\left(10^{\circ} \mathrm{C}\right)$. In this view, it is interesting to note the contrasting effect of cold storage on tomato compositional traits, resulting in a higher polyphenols accumulation in case of a lower lycopene content. This suggests the existence of a fine tuning among different classes of compounds in response to cold stress. However, by comparing the temporal trend of lycopene with that of its colorless precursors phytoene and phytofluene, clear time-dependent temperature effects on carotenogenesis where noticeable. Indeed, at $\mathrm{S}_{7}$, the highest lycopene content recorded at $20^{\circ} \mathrm{C}$ matched the strongest reduction of both phytoene and phytofluene. In other words, the lowest the lycopene concentration the highest the accumulation of its precursors and vice versa. This implies that reduced transformation kinetics of both phytoene and phytofluene represented the earliest metabolic constraints recorded in response to the imposed cold stress. According to Dumas et al. [53] the over-expression of phytoene desaturase (leading to lycopene synthesis by desaturating both phytoene and phytofluene) is the most important upstream metabolic step in increasing the lycopene content of tomato fruits at harvest. Our results bear this out in postharvest conditions too, as they indicate that, under mild cold stress storage conditions, desaturation of phytoene and phytofluene represents the earliest metabolic bottleneck in lycopene synthesis of cherry tomatoes, hence a possible priority target to modulate the postharvest evolution of their nutraceutical profile. On the other hand, to which extent this implies a mid-term modification of the overall nutraceutical profile of tomato represents an interesting topic, taking into account that, despite they are not effective antiradicals as lycopene, phytoene and phytofluene are among the prevailing carotenoids found in human plasma and tissues, and their bioaccessibility following gastro-intestinal digestion of tomato juice has been found $\sim 3-4$ fold higher than that of lycopene [54,55].

Among the studied cultivars 'Sugarland' proved the highest lycopene and total polyphenols content, whereas 'Eletta' overcame the other cultivars for phytoene and phytofluene. Excepting $\beta$-carotene, which over time increased more sharply in 'Eletta' and 'Ottymo', these differences were still noticeable at the end of the storage period, regardless of the storage temperature. This highlights, beyond the environmental influence, the existence of a strong genetic component determining the stoichiometric relationships among lycopene and its precursors. Unravelling the possible interactive effects among these three carotenoids in generating the antioxidative health benefits $[16,49]$ will allow for a better orientation of breeding programs toward the most convenient phytochemical evolution of tomatoes during refrigerated storage.

\section{Conclusions}

The results of the present experiment highlighted complex postharvest modifications of cherry tomatoes in response to the studied factors. By storing them under mild stressing conditions $\left(10^{\circ} \mathrm{C}\right)$ it was possible to improve the stability over time of carpometric traits (mainly fruit weight, firmness and Chroma) having commercial relevance, without alterations of compositional traits related to taste perception (reducing sugars content, SSC/TA and $\mathrm{pH}$ ). Moreover, when compared to $20^{\circ} \mathrm{C}$, storing at $10^{\circ} \mathrm{C}$ boosted the accumulation of total polyphenol and, at least in the short term (within 7 days of storage), the concentration of both phytoene and phytofluene, probably inhibiting their enzymatic desaturation leading to lycopene. This suggests their possible usefulness in modulating the nutraceutical evolution of cold stored cherry tomatoes during postharvest. This idea is reinforced by the stable varietal differences we found in terms of stoichiometric relationships among lycopene, phytoene and phytofluene. Regarding the varietal attitude to postharvest storage, the stability over time of fruit weight, dry matter content, SSC/TA and $\Delta E^{*}$ ab proved to be highly discriminant among cultivars, indicating the lowest ability of 'Ottymo' and 'Eletta' to maintain their fruit peculiarities over time. Thus, our results suggest the use of these variables to screen for cherry tomato germplasm suited to periods of postharvest storage. 
Author Contributions: Conceptualization, R.P.M., M.D. and E.A.; methodology, R.P.M., M.D. and S.B.; validation, F.G., R.P.M. and E.A.; formal analysis, M.D. and S.B.; investigation, M.D., R.P.M. and S.B.; data curation, M.D. and S.B.; writing—original draft preparation, R.P.M., E.A. and M.D.; writing—review and editing, F.G., C.L. and B.F.; supervision, F.G., C.L. and B.F. All authors have read and agreed to the published version of the manuscript.

Funding: This research received no external funding.

Conflicts of Interest: The authors declare no conflict of interest.

\section{References}

1. Food and Agriculture Organization of the United Nations. Available online: http://www.fao.org/faostat/en/ \#home (accessed on 26 September 2020).

2. Mauro, R.P.; Lo Monaco, A.; Lombardo, S.; Restuccia, A.; Mauromicale, G. Eradication of Orobanche/Phelipanche spp. seedbank by soil solarization and organic supplementation. Sci. Hortic. 2015, 193, 62-68. [CrossRef]

3. Capurso, C.; Vendemiale, G. The Mediterranean diet reduces the risk and mortality of the prostate cancer: A narrative review. Front. Nutr. 2017, 4, 38. [CrossRef] [PubMed]

4. Cainelli, N.; Ruperti, B. Biochemistry and molecular biology in fruits during cold storage. Annu. Plant Rev. Online 2019, 2, 659-688.

5. Mauro, R.P.; Agnello, M.; Rizzo, V.; Graziani, G.; Fogliano, V.; Leonardi, C.; Giuffrida, F. Recovery of eggplant field waste as a source of phytochemicals. Sci. Hortic. 2020, 261, 109023. [CrossRef]

6. Petric, T.; Kiferle, C.; Perata, P.; Gonzali, S. Optimizing shelf life conditions for anthocyanin-rich tomatoes. PLOS ONE 2018, 13, e0205650. [CrossRef]

7. Affandi, F.Y.; Verdonk, J.C.; Ouzounis, T.; Ji, Y.; Woltering, E.J.; Schouten, R.E. Far-red light during cultivation induces postharvest cold tolerance in tomato fruit. Postharvest Biol. Technol. 2020, 159, 111019. [CrossRef]

8. Tadesse, T.N.; Ibrahim, A.M.; Abtew, W.G. Degradation and formation of fruit color in tomato (Solanum lycopersicum L.) in response to storage temperature. Am. J. Food Technol. 2015, 10, 147-157. [CrossRef]

9. Farzaneh, V.; Gominho, J.; Pereira, H.; Carvalho, I.S. Screening of the antioxidant and enzyme inhibition ootentials of portuguese Pimpinella anisum L. seeds by GC-MS. Food Anal. Methods 2018, 11, 2645-2656. [CrossRef]

10. Park, M.H.; Sangwanangkul, P.; Baek, D.R. Changes in carotenoid and chlorophyll content of black tomatoes (Lycopersicon esculentum L.) during storage at various temperatures. Saudi J. Biol. Sci. 2018, 25, 57-65. [CrossRef]

11. Martí, R.; Roselló, S.; Cebolla-Cornejo, J. Tomato as a source of carotenoids and polyphenols targeted to cancer prevention. Cancers 2016, 8, 58. [CrossRef]

12. Story, E.N.; Kopec, R.E.; Schwartz, S.J.; Keith Harris, G. An update on the health effects of tomato lycopene. Annu. Rev. Food Sci. Technol. 2010, 1, 189-210. [CrossRef] [PubMed]

13. Peters, U.; Leitzmann, M.F.; Chatterjee, N.; Wang, Y.; Albanes, D.; Gelmann, E.P.; Friesen, M.D.; Riboli, E.; Hayes, R.B. Serum lycopene, other carotenoids, and prostate cancer risk: A nested case-control study in the prostate, lung, colorectal, and ovarian cancer screening trial. Cancer Epidemiol. Biomarkers Prev. 2007, 16, 962-968. [CrossRef] [PubMed]

14. Srivastava, S.; Srivastava, A.K. Lycopene; chemistry, biosynthesis, metabolism and degradation under various abiotic parameters. J. Food Sci. Technol. 2015, 52, 41-53. [CrossRef]

15. Liu, L.; Shao, Z.; Zhang, M.; Wang, Q. Regulation of carotenoid metabolism in tomato. Mol. Plant 2015, 8, 28-39. [CrossRef] [PubMed]

16. Gul, K.; Tak, A.; Singh, A.K.; Singh, P.; Yousuf, B.; Wani, A.A. Chemistry, encapsulation, and health benefits of beta-carotene-A review. Cogent Food Agric. 2015, 1. [CrossRef]

17. Basu, A.; Imrhan, V. Tomatoes versus lycopene in oxidative stress and carcinogenesis: Conclusions from clinical trials. Eur. J. Clin. Nutr. 2007, 61, 295-303. [CrossRef]

18. Engelmann, N.J.; Clinton, S.K.; Erdman, J.W.J. Nutritional aspects of phytoene and phytofluene, carotenoid precursors of lycopene. Adv. Nutr. 2011, 2, 51-61. [CrossRef]

19. Passam, H.; Karapanos, I.; Bebeli, P.; Savvas, D. A review of recent research on tomato nutrition, breeding and post-harvest technology with reference to fruit quality. Eur. J. Plant Sci. Biotechnol. 2007, 1, 1-21. 
20. Raiola, A.; Rigano, M.M.; Calafiore, R.; Frusciante, L.; Barone, A. Enhancing the health-promoting effects of tomato fruit for biofortified food. Mediators Inflamm. 2014, 2014, 1-16. [CrossRef]

21. Kavitha, P.; Shivashankara, K.S.; Rao, V.K.; Sadashiva, A.T.; Ravishankar, K.V.; Sathish, G.J. Genotypic variability for antioxidant and quality parameters among tomato cultivars, hybrids, cherry tomatoes and wild species. J. Sci. Food Agric. 2014, 94, 993-999. [CrossRef]

22. Alenazi, M.M.; Shafiq, M.; Alsadon, A.A.; Alhelal, I.M.; Alhamdan, A.M.; Solieman, T.H.I.; Ibrahim, A.A.; Shady, M.R.; Al-Selwey, W.A. Improved functional and nutritional properties of tomato fruit during cold storage. Saudi J. Biol. Sci. 2020, 27, 1467-1474. [CrossRef]

23. Mauro, R.P.; Agnello, M.; Distefano, M.; Sabatino, L.; San Bautista Primo, A.; Leonardi, C.; Giuffrida, F. Chlorophyll fluorescence, photosynthesis and growth of tomato plants as affected by long-term oxygen root zone deprivation and grafting. Agronomy 2020, 10, 137. [CrossRef]

24. Giuffrida, F.; Agnello, M.; Mauro, R.P.; Ferrante, A.; Leonardi, C. Cultivation under salt stress conditions influences postharvest quality and glucosinolates content of fresh-cut cauliflower. Sci. Hortic. 2018, 236, 166-174. [CrossRef]

25. Gautier, H.; Diakou-Verdin, V.; Bénard, C.; Reich, M.; Buret, M.; Bourgaud, F.; Poëssel, J.L.; Caris-Veyrat, C.; Génard, M. How does tomato quality (sugar, acid, and nutritional quality) vary with ripening stage, temperature, and irradiance? J. Agric. Food Chem. 2008, 56, 1241-1250. [CrossRef] [PubMed]

26. Khairi, A.N.; Falah, M.A.F.; Pamungkas, A.P.; Takahashi, N. Optimization of storage temperatures to maintain Lycopene content of tomato from moderate water stress irrigated greenhouse. IOP Conf. Ser. Mater. Sci. Eng. 2018, 403, 011001. [CrossRef]

27. Carli, P.; Barone, A.; Fogliano, V.; Frusciante, L.; Ercolano, M.R. Dissection of genetic and environmental factors involved in tomato organoleptic quality. BMC Plant Biol. 2011, 11, 58. [CrossRef]

28. McGuire, R.G. Reporting of objective color measurements. HortScience 1992, 27, 1254-1255. [CrossRef]

29. Atanasova, A.H.; Denev, P.N.; Tringovska, I.I.; Grozeva, S.Y.; Ganeva, D.G.; Kratchanova, M.G.; Panchev, I.N. Optimization of the key parameters for extraction of polyphenol compounds from tomato fruits (Solanum lycopersicum L.). Kinetics of the process. Bulg. Chem. Commun. 2014, 46, 65-70.

30. Gahler, S.; Otto, K.; Böhm, V. Alterations of vitamin C, total phenolics, and antioxidant capacity as affected by processing tomatoes to different products. J. Agric. Food Chem. 2003, 51, 7962-7968. [CrossRef]

31. Siracusa, L.; Patanè, C.; Rizzo, V.; Cosentino, S.L.; Ruberto, G. Targeted secondary metabolic and physico-chemical traits analysis to assess genetic variability within a germplasm collection of "long storage" tomatoes. Food Chem. 2018, 244, 275-283. [CrossRef]

32. Batu, A. Determination of acceptable firmness and colour values of tomatoes. J. Food Eng. 2004, 61, 471-475. [CrossRef]

33. Bui, H.T.; Makhlouf, J.; Ratti, C. Postharvest ripening characterization of greenhouse tomatoes. Int. J. Food Prop. 2010, 13, 830-846. [CrossRef]

34. Beckles, D.M. Factors affecting the postharvest soluble solids and sugar content of tomato (Solanum lycopersicum L.) fruit. Postharvest Biol. Technol. 2012, 63, 129-140. [CrossRef]

35. Tadesse, T.N.; Abtew, W.G. Effect of hot water treatment on reduction of chilling injury and keeping quality in tomato (Solanum lycopersicum L.) fruits. J. Stored Prod. Postharvest Res. 2016, 7, 61-68.

36. Leonardi, C.; Baille, A.; Guichard, S. Effects of fruit characteristics and climatic conditions on tomato transpiration in a greenhouse. J. Hortic. Sci. Biotechnol. 1999, 74, 748-756. [CrossRef]

37. Saladié, M.; Matas, A.J.; Isaacson, T.; Jenks, M.A.; Goodwin, S.M.; Niklas, K.J.; Xiaolin, R.; Labavitch, J.M.; Shackel, K.A.; Fernie, A.R.; et al. A reevaluation of the key factors that influence tomato fruit softening and integrity. Plant Physiol. 2007, 144, 1012-1028. [CrossRef]

38. Anton, D.; Bender, I.; Kaart, T.; Roasto, M.; Heinonen, M.; Luik, A.; Püssa, T. Changes in polyphenols contents and antioxidant capacities of organically and conventionally cultivated tomato (Solanum lycopersicum L.) fruits during ripening. Int. J. Anal. Chem. 2017, 2017, 6-10. [CrossRef]

39. Mauro, R.P.; Rizzo, V.; Leonardi, C.; Mazzaglia, A.; Muratore, G.; Distefano, M.; Sabatino, L.; Giuffrida, F. Influence of harvest stage and rootstock genotype on compositional and sensory profile of the elongated tomato cv. Sir Elyan. Agriculture 2020, 10, 82. [CrossRef]

40. Ganje, M.; Jafari, S.M.; Farzaneh, V.; Malekjani, N. Kinetics modelling of color deterioration during thermal processing of tomato paste with the use of response surface methodology. Heat Mass Transf. 2018, 54, 3663-3671. [CrossRef] 
41. Clément, A.; Dorais, M.; Vernon, M. Multivariate approach to the measurement of tomato maturity and gustatory attributes and their rapid assessment by vis-NIR spectroscopy. J. Agric. Food Chem. 2008, 56, 1538-1544. [CrossRef]

42. Goisser, S.; Wittmann, S.; Fernandes, M.; Mempel, H.; Ulrichs, C. Comparison of colorimeter and different portable food-scanners for non-destructive prediction of lycopene content in tomato fruit. Postharvest Biol. Technol. 2020, 167, 111232. [CrossRef]

43. Licciardello, F.; Lombardo, S.; Rizzo, V.; Pitino, I.; Pandino, G.; Strano, M.G.; Muratore, G.; Restuccia, C.; Mauromicale, G. Integrated agronomical and technological approach for the quality maintenance of ready-to-fry potato sticks during refrigerated storage. Postharvest Biol. Technol. 2018, 136, 23-30. [CrossRef]

44. Dattner, M.; Bohn, D. Characterization of print quality in terms of colorimetric aspects. In Printing on Polymers: Fundamentals and Applications; Elsevier Inc.: Amsterdam, The Netherlands, 2015; pp. 329-345. ISBN 9780323374682.

45. Fernandes, I.; Leça, J.M.; Aguiar, R.; Fernandes, T.; Marques, J.C.; Cordeiro, N. Influence of crop system fruit quality, carotenoids, fatty acids and phenolic compounds in cherry tomatoes. Agric. Res. 2020. [CrossRef]

46. Mapelli-Brahm, P.; Stinco, C.M.; Meléndez-Martínez, A.J. Comparative study of the bioaccessibility of the colorless carotenoids phytoene and phytofluene in powders and pulps of tomato: Microstructural analysis and effect of addition of sunflower oil. Food Funct. 2018, 9, 5016-5023. [CrossRef] [PubMed]

47. Sharma, A.; Shahzad, B.; Rehman, A.; Bhardwaj, R.; Landi, M.; Zheng, B. Response of phenylpropanoid pathway and the role of polyphenols in plants under abiotic stress. Molecules 2019, 24, 2452. [CrossRef]

48. Holst, B.; Williamson, G. Nutrients and phytochemicals: From bioavailability to bioefficacy beyond antioxidants. Curr. Opin. Biotechnol. 2008, 19, 73-82. [CrossRef]

49. Mirdehghan, S.H.; Valero, D. Bioactive compounds in tomato fruit and its antioxidant activity as affected by incorporation of Aloe, eugenol, and thymol in fruit package during storage. Int. J. Food Prop. 2017, 20, 1798-1806.

50. Brashlyanova, B.P.; Pevicharova, G.T. Effects of cold storage and ripening on antioxidant components in tomatoes. Acta Hortic. 2009, 830, 349-353. [CrossRef]

51. Rodriguez-Amaya, D.B. Nature and distribution of carotenoids in foods. In Shelf Life Studies of Foods and Beverages Chemical, Biological, Physical and Nutritional Aspects; Charalambous, G., Ed.; Elsevier Science: Amsterdam, The Netherlands, 1993; ISBN 0-444-89459-4.

52. Gruszecki, W.I.; Strzałka, K. Carotenoids as modulators of lipid membrane physical properties. Biochim. Biophys. Acta Mol. Basis Dis. 2005, 1740, 108-115. [CrossRef]

53. Dumas, Y.; Dadomo, M.; Di Lucca, G.; Grolier, P. Effects of environmental factors and agricultural techniques on antioxidant content of tomatoes. J. Sci. Food Agric. 2003, 83, 369-382. [CrossRef]

54. Meléndez-Martínez, A.J.; Mapelli-Brahm, P.; Benítez-González, A.; Stinco, C.M. A comprehensive review on the colorless carotenoids phytoene and phytofluene. Arch. Biochem. Biophys. 2015, 572, 188-200. [CrossRef] [PubMed]

55. Mapelli-Brahm, P.; Corte-Real, J.; Meléndez-Martínez, A.J.; Bohn, T. Bioaccessibility of phytoene and phytofluene is superior to other carotenoids from selected fruit and vegetable juices. Food Chem. 2017, 229, 304-311. [CrossRef] [PubMed]

Publisher's Note: MDPI stays neutral with regard to jurisdictional claims in published maps and institutional affiliations.

(C) 2020 by the authors. Licensee MDPI, Basel, Switzerland. This article is an open access article distributed under the terms and conditions of the Creative Commons Attribution (CC BY) license (http://creativecommons.org/licenses/by/4.0/). 